\title{
Advances in soft-tissue sarcoma - There are no mistakes, only lessons to learn!
}

\author{
Sameer Rastogi, Parisa Manasa', Aditi Aggarwal'², Kaushal Kalra
}

\begin{abstract}
Background: In this decade the treatment of advanced sarcoma has seen many highs and lows in terms of successful trials and failed trials. This is possible due to great collaborations, newer therapies and histology focused trials. Methods: In ASCO 2019 many sarcoma trials were presented and we chose 3 challenging clinical trials that widen our perspective on soft tissue sarcoma. We have critically analyzed the data and have discussed the implications of these trials on current practice. First trial was ANNOUNCE trial which was done to confirm the efficacy of olaratumab after its dramatic success in advanced soft tissue sarcoma in a phase 2 trial.Another trial STRASS trial, which was unique because of being first successfully conducted randomized trial addressing preoperative radiotherapy in retroperitoneal soft tissue sarcoma. Third trial was phase 2 trial SARC 028 trial exploring the role of immunotherapy in pleomorphic undifferentiated sarcoma and liposarcoma subgroup. Result: ANNOUNCE trial failed to show OS benefit in olaratumab/doxorubicin arm as compared to doxorubicin/placebo arm . Based upon this FDA has revoked the approval of olaratumab leading to nihilism and disappointment amongst oncologists. In STRASS trial failed to meet the primary end point though there was a benefit in the liposarcoma subgroup in terms of abdominal recurrence free survival. There are several reasons that this trial might have failed. First, RPSs are not homogeneous population. RPSs might behave very differently as per the histopathology ranging from well differentiated LPS to leiomyosarcoma. Since the event rate in well-differentiated liposarcoma might happen late, the median follow-up of 43 months might not be sufficient. In SARC trial ORR in pleomorphic undifferentiated sarcoma (PUS) cohort was $9 / 40$ (22.5\%), while response rates in liposarcoma cohort were $4 / 39$ (I0.2\%). There was poor correlation between the response and the tumor cells' PD-LI positivity. Simultaneously, we must not take for granted the role of pembrolizumab in PUS as the previous study (PEMBROSARC) had also showed dismal outcomes with immunotherapy. Conclusion: In this paper we discuss the intricacies of these trials and how they affect the rapidly changing landscape in advanced soft tissue sarcoma.

Key words: ASCO, Soft tissue sarcoma, STS, Leiomyosarcoma, Relapse, LPS, PUS, Olaratumab, Pembrolizumab
\end{abstract}

Every other year the field of sarcoma is taking big leaps in terms of collaborations and the efforts to make the difference. This year, three major trials (practice changing or not so practice changing) were presented for soft-tissue sarcoma (STS), namely Announce trial, SARC 028 trial, and STRASS trial.

Olaratumab was approved in October 2016 based on Phase $1 \mathrm{~b} / 2$ trial showing the dramatic overall survival (OS) benefit of 11.8 months which was unprecedented leading to both reassurance and speculations after the Food and Drug Administration (FDA) accelerated approval. ${ }^{[1]}$ It was followed by ANNOUNCE trial, a phase 3 placebo control trial presented in plenary session. ${ }^{[2]}$ This trial had OS as a primary endpoint OS in overall population along with co-primary endpoint as OS in leiomyosarcoma subgroup. This trial failed to show OS benefit in olaratumab/doxorubicin arm as compared to doxorubicin/placebo arm (20.4 vs. 19.7 months, $P=0.69)$. While this has led to overt disappointment among patient advocates and sarcoma oncologists, there are quite a few lessons learned from this trial. Another point of discussion was improved OS in the comparator arm which is apparently more than previous first-line trials (though one-fourth of patients in this study were second line!). Hence, what could lead to these disparate results in Phase 1b/2 trial as compared to Phase 3 trial.

First, olaratumab approval was based on Phase 2 randomized trial which had higher chances of error. Second, in all sarcoma trials, there is a significant percentage of "other sarcomas" population, the affect of which on outcome is totally unknown. Third, with such failure, the concept of FDA accelerated approval (approving drug based on surrogate endpoint and then confirming in confirmatory endpoint) comes under scrutiny. A recent analysis done by Gyawali et al. showed that only $19 \%$ of FDA accelerated approved drugs could be tested with confirmatory endpoint such as OS. ${ }^{[3]}$ Improved OS in the

\begin{tabular}{|l|}
\hline Access this article online \\
\hline Quick Response Code: \\
\\
Website: www.sajc.org \\
\hline DOI: 10.4103 /sajc.sajc_215_19 \\
\hline
\end{tabular}

comparator arm could be because of better supportive care and more use of second/third-line therapies.

STRASS trial was a randomized, multicenter trial in patients with histologically proven localized retroperitoneal sarcoma (RPS) which was operable and suitable for preoperative radiotherapy (RT) ${ }^{[4]}$ This study was done in view of a higher risk of local recurrence in RPSs and previous nonrandomized studies, showing survival benefit in RPS. ${ }^{[5]}$ This study succeeded partly as it could complete its accrual unlike previous ACOSOG trial which failed to complete accrual. The primary endpoint in this study was abdominal relapse free survival which is shown in Table 1.

There were a total of 266 patients with majority of patients were liposarcoma (75\%) followed by leiomyosarcoma (14\%) and others. Majority of the patients received intensity-modulated RT $n=95 \%$, while 5\% received three-dimensional conformal radiation therapy.

Macroscopic surgery was done in $95 \%$ in both arms. After a median follow-up of 43 months, the abdominal relapse free survival at 3 years in preoperative RT arm versus surgery arm was $60.4 \%$ versus $58.7 \%$. Three years' OS in both the arms was $84 \%$ versus $84.6 \%$, suggesting that only a few life-threatening events would have happened during this follow-up. After adjusting the (Independent Data Monitoring Committee) IDMC-recommended modification (stated above), there was a benefit in the liposarcoma subgroup in terms of abdominal recurrence free survival.

There are several reasons that this trial might have failed. First, RPSs are not homogeneous population. RPSs might behave very differently as per the histopathology ranging from well differentiated (5 year local recurrence rate $-<15 \%-20 \%$ and distant metastasis $-<2 \%$ ) to leiomyosarcomas (distance metastasis $-56 \%$ and local recurrence $-10 \%-20 \%){ }^{[6]}$ Besides well-differentiated RPS, local recurrences happen at the rate of $4 \%$ per year. It might

This is an open access journal, and articles are distributed under the terms of the Creative Commons Attribution-NonCommercial-ShareAlike 4.0 License, which allows others to remix, tweak, and build upon the work non-commercially, as long as appropriate credit is given and the new creations are licensed under the identical terms. Society, New Delhi, ${ }^{2}$ Department of Radiation Oncology, AlIMS, NCl, Jhajjar, Haryana, India Correspondence to: Dr. Sameer Rastogi, E-mail:samdoc_mamc@yahoo.com
For reprints contact: reprints@medknow.com

How to cite this article: Rastogi S, Manasa P, Aggarwal A, Kalra K. Advances in soft-tissue sarcoma - There are no mistakes, onlylessons to learn! South Asian J Cancer 2019;8:258-9. 
Table 1: Definition of Abdominal relapse free survival

\begin{tabular}{ll}
\hline After surgery & Before surgery \\
\hline Local relapse after macroscopic & Tumor became inoperable between \\
complete resection & randomization and time of surgery \\
Peritoneal sarcomatosis found & $\begin{array}{l}\text { Development of distant metastasis } \\
\text { while on preoperative RT }\end{array}$ \\
at laparotomy & Local progression of primary \\
Macroscopic local disease left & tumor while on preoperative RT \\
behind at laparotomy & The IDMC recommended on July 2017 to remove local progression while on RT as \\
\hline The & endpoint in patients who could undergo surgery. RT=Radiotherapy
\end{tabular}

be therefore understandable that because of the high rate of local recurrence liposarcoma is the only subtype which could be benefited by preoperative RT as shown in subgroup analysis. Since the event rate in well-differentiated liposarcoma might happen late, the median follow-up of 43 months might not be sufficient. In such a collaborative trial with transatlantic collaboration, it is also pertinent to do operability assessment of this heterogeneous group centrally.

Regarding the third trial which was a poster at American Society of Clinical Oncology (ASCO) meeting, it was one of the most awaited studies to evaluate the effect of immunotherapy (pembrolizumab) in STSs. SARC 028 study was a Phase 2 trial, in which 10 patients each of pleomorphic undifferentiated sarcoma, leiomyosarcoma, synovial sarcoma, and liposarcoma (total of 40 patients) were given pembrolizumab every 3 weekly ${ }^{[7]}$ Of 40 patients, 7 (17\%) patients had objective responses. Responses in this were mainly limited to pleomorphic undifferentiated sarcoma $(4 / 10=40 \%)$ and liposarcoma $(2 / 10=20 \%)$. Based on this, it was apparent that it should be further studied in pleomorphic undifferentiated sarcoma and liposarcoma. SARC 028 expansion cohort included further 30 patients each of pleomorphic undifferentiated sarcoma and liposarcoma. ${ }^{[8]}$ It was assumed that if the overall response rate (ORR) exceeds $25 \%$ in the respective type of sarcoma, it is clinically meaningful. ORR in pleomorphi undifferentiated sarcoma (PUS) cohort was 9/40 (22.5\%), while response rates in liposarcoma cohort were $4 / 39(10.2 \%)$. Thus, it was concluded that since it was just short of $25 \%$ in PUS, this might be the subset in which this therapy might work. In patients with PUS those who responded $(N=9)$, majority of patients $7 / 9(78 \%)$, the responses lasted $<20$ weeks. Furthermore, programmed death-ligand 1 (PD-L1) testing in tumor cells was also done to find the predictive biomarkers and was considered positive if it was $>1 \%$. There was poor correlation between the response and the tumor cells' PD-L1 positivity. Of a total of 11 patients who responded, PD-L1 was positive in 6 patients. Furthermore, 13 nonresponders were PD-L1 positive. This reiterates the fact that immune cells' PD-L1 might play a more important role than tumor cells' PD-L1. ${ }^{[9]}$ Simultaneously, we must not take for granted the role of pembrolizumab in PUS as the previous study showed dismal outcomes. ${ }^{[9]}$ Hence, future studies with more patient numbers and better design are definitely required to answer this question.

In nutshell, these three trials tried to answer very relevant questions in this extremely rare disease. The collaboration and conduct of randomized trials seem to be the way ahead. The future certainly belongs to histology-specific answers and multinational and multicenter trials.

\section{References}

1. Tap WD, Jones RL, Van Tine BA, Chmielowski B, Elias AD, Adkins D, et al. Olaratumab and doxorubicin versus doxorubicin alone for treatment of soft-tissue sarcoma: An open-label phase $1 \mathrm{~b}$ and randomised phase 2 trial. Lancet 2016;388:488-97.

2. Tap WD, Wagner AJ, Papai Z, Ganjoo KN, Yen CC, Schoffski P, et al. Announce: A randomized, placebo (PBO)-controlled, double-blind, phase (Ph) III trial of doxorubicin (dox) + olaratumab versus dox + PBO in patients (pts) with advanced soft tissue sarcomas (STS). J Clin Oncol 2019;37 18 Supp I:LBA3.

3. Assessment of the Clinical Benefit of Cancer Drugs Receiving Accelerated Approval | Oncology | JAMA Internal Medicine | JAMA Network. Available from: https://jamanetwork.com/journals/jamainternalmedicine / fullarticle/2733561 [Last accessed on 2019 Jul 09].

4. Bonvalot S, Gronchi A, Pechoux CL, Swallow CJ, Dirk C, Strauss, et al. STRASS (EORTC 62092): A phase III randomized study of preoperative radiotherapy plus surgery versus surgery alone for patients with retroperitoneal sarcoma. J Clin Oncol 2019;37:11001. Available from: https://ascopubs.org/doi/abs/10.1200/ JC0.2019.37.15_suppl.11001. [Last accessed on 2019 Aug 30].

5. Nussbaum DP, Rushing CN, Lane WO, Cardona DM, Kirsch DG, Peterson $\mathrm{BL}$, et al. Preoperative or postoperative radiotherapy versus surgery alone for retroperitoneal sarcoma: A case-control, propensity score-matched analysis of a nationwide clinical oncology database. Lancet Oncol 2016; 17:966-75.

6. Dingley B, Fiore M, Gronchi A. Personalizing surgical margins in retroperitoneal sarcomas: An update. Expert Rev Anticancer Ther 2019; 19:613-31.

7. Tawbi HA, Burgess M, Bolejack V, Van Tine BA, Schuetze SM, Hu J, et al. Pembrolizumab in advanced soft-tissue sarcoma and bone sarcoma (SARC028): A multicentre, two-cohort, single-arm, open-label, phase 2 trial. Lancet Oncol 2017; 18:1493-501.

8. Burgess MA, Bolejack V, Schuetze S, Van Tine BA, Attia S, Riedel RF, et al. Clinical activity of pembrolizumab $(P)$ in undifferentiated pleomorphic sarcoma (UPS) and dedifferentiated/pleomorphic liposarcoma (LPS): Final results of SARC028 expansion cohorts. J Clin Oncol 2019;3715 supp I: 11015.

9. Toulmonde M, Italiano A. PD-1 inhibition in sarcoma still needs investigation. Lancet Oncol 2018;19:e6. 